\title{
Multistage Frequency-Hopping Assisted Ultra-Wideband Multiple-Access Communications
}

\author{
Lie-Liang Yang and Lajos Hanzo \\ Dept. of Electronics and Computer Sciences \\ University of Southampton, SO17 1BJ, UK. \\ Tel: +44-703-593 125, Fax: +44-703-594 508
}

\begin{abstract}
Ultra-WideBand (UWB) systems having a bandwidth on the order of a Gigahertz have recently received wide attention both in the USA and in Europe. In this contribution a novel UWB multiple access scheme based on the well-known family of Frequency-Hopping Multiple Access (FHMA) techniques is proposed, where the associated FH is implemented using MultiStage Frequency Hopping Multiple Access, or MS/UWB FHMA. We highlight the principles of the proposed MS/UWB FHMA communication system, investigate the associated spectrum assignment, the FH strategy, the applicable signal detection schemes as well as the achievable Bit Error Rate (BER) performance. Our study shows that MS/UWB FHMA is capable of supporting an extremely high number of users, while employing relatively simple receivers.
\end{abstract}

\section{INTRODUCTION}

The last decade has witnessed a paradigm shift from lowbitrate narrowband wireless systems to broadband wireless systems. This trend continued with the recent emergence of the family of Ultra-WideBand (UWB) systems [1] - [5], also referred to as impulse radio or time domain radio systems, which have received considerable attention both in the USA and in Europe. The first UWB propagation results were reported in [1]. It was shown in [1] - [3] that UWB signals do not suffer from severe fading, since they benefit from the presence of a huge number multipath components. Therefore the performance of UWB systems is robust against the hostile channel-induced impairments of conventional narrower bandwidth systems.

In this contribution an alternative multiple access scheme is proposed, which allows each user to access an ultra-wideband. The proposed UWB multiple access scheme is based on multistage Frequency-Hopping (FH) invoked in the context of noncoherent $M$-ary Frequency Shift Keying (MFSK). Hence we refer to the proposed scheme as MultiStage Ultra-WideBand Frequency-Hopping Multiple Access and use the abbreviation of MS/UWB FHMA. In this contribution we highlight the novel concept of MS/UWB FHMA. The spectrum assignment and FH strategy, the system design parameters as well as the Bit Error Rate (BER) performance associated with different detection philosophies are studied. Our investigations will demonstrate that MS/UWB FHMA constitutes a high-efficiency multiple access scheme capable of supporting an extremely high number

This work has been funded in the framework of the IST project IST-199912070 TRUST, which is partly funded by the European Union. The authors would like to acknowledge the contributions of their colleagues.

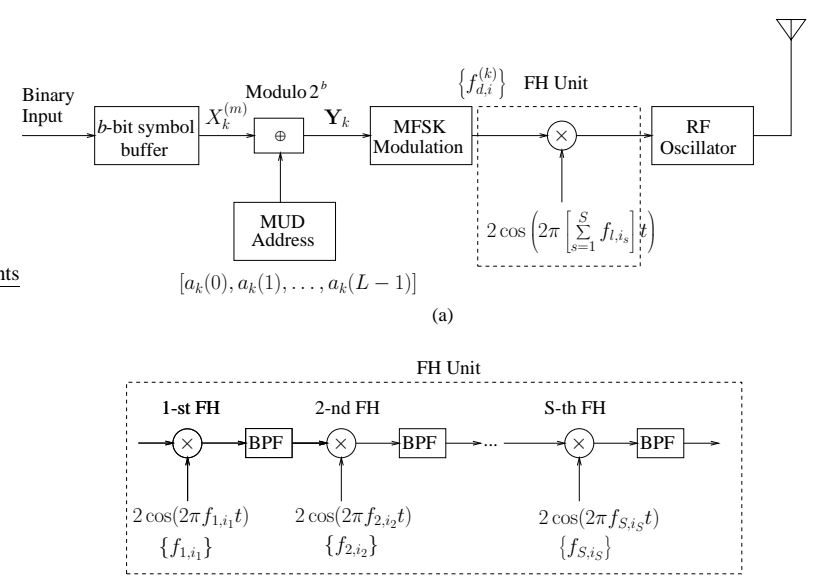

(b)

Fig. 1. Transmitter block diagram of the MS/UWB FHMA system using MFSK modulation.

of users with the aid of relatively simple receivers previously proposed in the literature[6] - [8].

\section{SySTEM DESCRIPTION}

\section{A. Transmitter}

The operation of the transmitter associated with the MS/ UWB FHMA system studied may be understood by referring to Fig.1. During a signalling interval of $T_{s}$ duration, $b$ message bits of the $k$ th user having a bit rate of $R_{b}$ are loaded into a $b$-bit buffer. We denote this $b$-bit symbol as $X_{k}^{(m)}$, where $X_{k}^{(m)} \in\left[0, M=2^{b}\right)$. Hence, the $M=2^{b}$-ary output symbol rate is $R_{s}=R_{b} / b$. Let $R_{h}=1 / T_{h}$ be the FH rate, where $T_{h}$ represents the duration of the $\mathrm{FH}$ tones or chips. The ratio of $L=T_{s} / T_{h}$ determines, whether slow FH (SFH) - where $L<1$ - or fast FH (FFH) - where $L \geq 1$ - is invoked. Specifically, if $L<1$, several $M=2^{b}$-ary symbols are transmitted in a FH-interval of $T_{h}$ duration, activating one out of $M$ legitimate frequencies of an MFSK scheme. By contrast, if $L \geq 1$ and integer, an $M=2^{b}$-ary symbol activates a particular MFSK tone only for the duration of $T_{h}=T_{s} / L$ seconds and then hops to a further $(L-1)$ other legitimate frequencies of the MFSK scheme used.

In our proposed system we assume that $L=T_{s} / T_{h}$ is an integer larger than one, i.e. that FFH is employed [6]. In order 
to distinguish between different users, each user is assigned a user-signature FH pattern also referred to as an address code [6] - [8]. Let the address code of the $k$ th user, $1 \leq k \leq K$, be expressed as $\mathbf{a}_{k}=\left[a_{k}(0), a_{k}(1), \ldots, a_{k}(L-1)\right]$, where $a_{k}(l) \in G F(M), l=0,1, \ldots, L-1$ and $G F(M)$ denotes a Galois field over $M=2^{b}$. Then, the transmitted symbol $X_{k}^{(m)}$ is signalled by invoking the $k$ th user's address code, which can be expressed as

$$
\mathbf{Y}_{k}=\left[y_{k}(0), y_{k}(1), \ldots, y_{k}(L-1)\right]=X_{k}^{(m)} \cdot \mathbf{1} \oplus \mathbf{a}_{k},
$$

where 1 is a unit vector of length $L, y_{k}(l)$ for $1 \leq l<L$ are elements of $G F(M)$, and $\oplus$ denotes modulo- $M$ or modulo$2^{b}$ addition, as seen in Fig.1. Obeying the user signature, the components of $\mathbf{Y}_{k}$ are passed serially to an MFSK modulator, where they are converted to the MFSK tones $\mathbf{F}_{m}^{(k)}=$ $\left[f_{d, 0}^{(k)}, f_{d, 1}^{(k)}, \ldots, f_{d, L-1}^{(k)}\right]$ referred to as chips of duration $T_{h}$. Following MFSK modulation FH is activated obeying the userspecific $\mathrm{FH}$ pattern derived using the multistage $\mathrm{FH}$ scheme to be detailed in the next Subsection during our forthcoming discourse. Finally, the modulated signal is transmitted using the RF mixing stage. During the time interval $t \in\left[i T_{s},(i+1) T_{s}\right]$, the $k$ th user's transmitted signal can be expressed as

$$
\begin{aligned}
s_{k}^{(i)}(t)= & \sum_{l=0}^{L-1} \sqrt{\frac{2 P}{L}} p_{T_{h}}\left(t-i T_{s}-l T_{h}\right) \\
& \cdot \cos \left(2 \pi\left[\sum_{s=1}^{S} f_{s, i_{s}, l}^{(k)}+f_{d, l}^{(k)}\right] t+\psi_{i, l}^{(k)}\right),
\end{aligned}
$$

where $P$ is the transmitted power, $p_{T_{h}}(t)$ is the pulse-shaped waveform associated with a chip defined over the interval $\left[0, T_{h}\right), S$ represents the number of FH stages seen in Fig.1(b), and $f_{s, i_{s}, l}^{(k)}$ represents the base frequency corresponding to the $i$ th transmitted symbol in the $s$ th FH stage, $s=1,2, \ldots, S$ during the $l$ th chip interval. Finally, $\psi_{i, l}^{(k)}$ represents the phase introduced by the MFSK modulation and $\mathrm{FH}$ procedures.

The concept of multistage FH can be augmented with the aid of Fig.1(b) and Fig.2. As an example, we characterize a three-stage FHMA system associated with 16-ary MFSK modulation, which will be further detailed during our forthcoming discourse. In the MS/UWB FHMA system the total bandwidth of $W_{s s}$ is first uniformly divided into $m_{S}$ number of frequency bands, each having a bandwidth of $W_{F H}^{S}=W_{s s} / m_{S}$ - which is referred to as the Stage- $S$ subband - and each subband is correspondingly assigned a frequency from the Stage$S$ frequency set expressed as $\mathbf{F}_{S}=\left[f_{S, 0}, f_{S, 1}, \ldots, f_{S, m_{S}-1}\right]$. In the example of Fig.2 this corresponds to Stage-3 at the top. Then, the Stage- $S$ subband is further divided, again, uniformly into $m_{S-1}$ number of frequency bands, where each band has a bandwidth of $W_{F H}^{S-1}=W_{F H}^{S} / m_{S-1}$. We refer to $W_{F H}^{S-1}$ as the Stage- $(S-1)$ subband, which is Stage2 in the example of Fig.2. Following the procedure used at Stage- $S$, each of the Stage- $(S-1)$ subbands is assigned a frequency from the Stage- $(S-1)$ frequency set expressed as

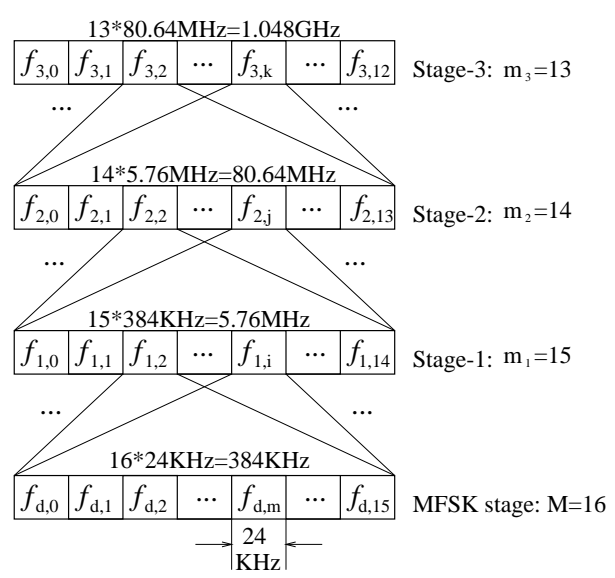

Fig. 2. Example of the spectrum allocation of a 3-stage $(L=3)$ FH SSMA system using 16-ary MFSK modulation, where $m_{1}=15, m_{2}=14, m_{3}=$ 13 and $R_{b}=32 \mathrm{~Kb} / \mathrm{s}$.

$\mathbf{F}_{(S-1)}=\left[f_{(S-1), 0}, f_{(S-1), 1}, \ldots, f_{(S-1), m_{(S-1)}-1}\right]$. According to the above rules, finally, the Stage- 2 subband is uniformly divided into $m_{1}$ number of frequency bands, each having a bandwidth of $W_{F H}^{1}=W_{F H}^{2} / m_{1}$, which are referred to as the Stage-1 subbands. At the same time, each of the Stage-1 subbands is assigned a frequency from the Stage- 1 frequency set of $\mathbf{F}_{1}=\left[f_{1,0}, f_{1,1}, \ldots, f_{1, m_{1}-1}\right]$.

Based on the above bandwidth assignment, the total number of FH bands is $M_{t}=\prod_{s=1}^{S} m_{s}$. As shown in Fig.2, each FH band consists of $M$ tones associated with MFSK modulation. We referred to this processing stage as the MFSK stage. Since the FH rate is $R_{h}$, the frequency spacing between two adjacent MFSK tones should be no less than $R_{h}$, which is an often-used system constraint in FFH [6]. Consequently, the total system bandwidth $W_{s s}$ and the $\mathrm{FH}$ rate $R_{h}$ must obey the relationship of $W_{s s} \geq \prod_{s=1}^{S} m_{s} \times M R_{h}$. Since we have $R_{h}=L R_{s}=L R_{b} / b$, where $R_{s}$ and $R_{b}$ are the symbol rate and bit rate, respectively, the relationship of $W_{s s} \geq \prod_{s=1}^{S} m_{s} \times M L R_{b} / b$ holds. We assume that the FH rate at each FH stage is $R_{H}=1 / T_{H}$, i.e. the frequencies at a given FH stage $s, s=1,2, \ldots, S$ hop $\omega=T_{s} / T_{H}$ times per $M=2^{b}$-ary symbol. For example, $\omega=1$ would imply that the FH rate $R_{H}$ at each FH stage is the same as the symbol rate $R_{s}$, i.e. $R_{H}=R_{s}$. Furthermore, we assume that the relative FH delay between two adjacent $\mathrm{FH}$ stages is $T_{H} / S$, i.e. that the $\mathrm{FH}$ at the $(s+1)$ th stage happens $T_{H} / S$ seconds earlier or later than that at the $s$ th stage. Consequently, the actual number of hops (chips) per symbol, $L$, the number of hops, $\omega$, at each FH stage, as well as the number of FH stages, $S$, obey the relationship of $L=T_{s} /\left(T_{H} / S\right)=\omega S$.

Based on the above spectrum assignment strategy and assumptions, multistage FH can be implemented as follows. After the MFSK modulation stage of Fig.1, each MFSK tone is frequency-hopped using $S$ number of possible frequencies from the $S$ frequency sets, one from each set, according to the $\mathrm{FH}$ strategy to be outlined in the next subsection. The FH frequency to be activated during each of the $L$ chips is determined by the sum of the frequencies activated at the $S$ number of FH stages, 
i.e. by $\sum_{s=1}^{S} f_{s, i_{s}, l}$, as shown in the Fig.1.

\section{B. Residue Number System Based FH}

It is widely recognized that in FHMA communications the FH patterns must be assigned such that: a) there is no ambiguity concerning the identity of the user and the information it transmits; b) the probability of a hit or collision between the frequencies activated by any two FH patterns is as low as possible, which implies that each user must interfere as small a fraction of time as possible with the other users; c) all frequencies in the FH band are activated with the same probability; and d) it is also desirable for the transmitter to hop to a frequency far from the previous one, i.e. the consecutively activated MFSK tones of the FH patterns must be spaced sufficiently far apart, so that the channel's characteristics associated with consecutive hops become independent. In this Subsection a FH scheme based on the Residue Number System (RNS) suitable for the proposed synchronous multistage FHMA system is contrived. Let us first give a brief introduction to the basic philosophy of RNSs.

A RNS [9] is defined by the choice of $S$ positive integers $m_{1}, m_{2}, \ldots, m_{S}$ referred to as moduli. If all the moduli are pairwise relative primes, any integer $\mathbf{A}_{k}$ - which is used to describe the $k$ th user's FH address, can be uniquely and unambiguously represented by the residue sequence $\mathbf{A}_{k} \Leftrightarrow$ $\left(A_{k 1}, A_{k 2}, \ldots, A_{k S}\right)$ in the range of $0 \leq \mathbf{A}_{k}<M_{S}$, where $A_{k s}=\mathbf{A}_{k}\left(\bmod m_{s}\right)$ represents the residue digits of $\mathbf{A}_{k}$ upon division by $m_{s}$, and $M_{S}=\prod_{s=1}^{S} m_{s}$ is the dynamic range of the RNS. By contrast, the notation $\mathbf{a}_{k}$ will be used in the context of multiuser detection to be introduced in Section III, which will be referred to as the $k$ th user's multiuser detection (MUD) address. According to the Chinese Remainder Theorem (CRT) [9], for any given $S$-tuple $\left(A_{k 1}, A_{k 2}, \ldots, A_{k S}\right)$, where $0 \leq A_{k s}<m_{s}$, there exists one and only one integer $\mathbf{A}_{k}$ such that $0 \leq \mathbf{A}_{k}<M_{S}$ and $A_{k s}=\mathbf{A}_{k}\left(\bmod m_{s}\right)$, which allows us to uniquely recover the integer value of $\mathbf{A}_{k}$ from the received residue digits. Again, $\mathbf{A}_{k}$ represents the $\mathrm{FH}$ address of user $k$.

Assuming that the integers $\mathbf{A}_{\mathbf{1}}$ and $\mathbf{A}_{\mathbf{2}}$ have RNS representations of $\mathbf{A}_{\mathbf{1}} \Leftrightarrow\left(A_{11}, A_{12}, \ldots, A_{1 S}\right)$ and $\mathbf{A}_{\mathbf{2}} \Leftrightarrow\left(A_{21}, A_{22}\right.$, $\left.\ldots, A_{2 S}\right)$, respectively, then $\mathbf{A}_{\mathbf{1}} \bullet \mathbf{A}_{\mathbf{2}}$, where $\bullet$ denotes addition, subtraction or multiplication, yields another unique residue sequence $\mathbf{A}_{\mathbf{3}}$. The arithmetic operations over the RNS can be carried out on a residue-by-residue basis, which can be expressed as [9]:

$$
\mathbf{A}_{\mathbf{3}}=\mathbf{A}_{\mathbf{1}} \bullet \mathbf{A}_{\mathbf{2}}\left(\bmod M_{S}\right) \Leftrightarrow\left[\left(A_{1 s} \bullet A_{2 s}\right)\left(\bmod m_{s}\right)\right]_{s=1}^{S},
$$

where on the left of (3) the operation $\bullet$ represents modulo- $M_{S}$ addition, subtraction or multiplication of two integers, and at the right of (3) • represents the same operations performed on the basis of the appropriate residue digits $A_{1 s}$ and $A_{2 s}$, with respect to their corresponding modulus $m_{s}$.

Single-User FH: The basic principle of the RNS-based multistage FH scheme is that the frequencies at the $S$ number of stages are activated according to the $S$ number of residue digits of a corresponding residue sequence in the RNS. Specifically, let $\mathbf{A}_{k}^{i} \Leftrightarrow\left(A_{k 1}^{i}, A_{k 2}^{i}, \ldots, A_{k S}^{i}\right)$ be the residue sequence representation of integer $\mathbf{A}_{k}^{i}$ in terms of a RNS using moduli $m_{1}, m_{2}, \ldots, m_{S}$, where the superscript $i$ represents the time index. Then the FH frequency at instant $i$ is determined by the sum of $f_{1, A_{k 1}^{i}}$ from the Stage-1 frequency set $\mathbf{F}_{1}, f_{2, A_{k 2}^{i}}$ from the Stage- 2 frequency set $\mathbf{F}_{2}$, an so on until $f_{S, A_{k S}^{i}}$ from the Stage- $S$ frequency set $\mathbf{F}_{S}$, i.e. by $\sum_{s=1}^{S} f_{s, A_{k s}^{i}}$. At the time index $(i+1), \mathbf{A}_{k}^{i}$ is increased by one and hence it is represented in the RNS domain as $\mathbf{A}_{k}^{(i+1)}=\mathbf{A}_{k}^{i}+1 \Leftrightarrow$ $\left(A_{k 1}^{(i+1)}, A_{k 2}^{(i+1)}, \ldots, A_{k S}^{(i+1)}\right)$. Similarly, the FH frequency at instant $(i+1)$ is determined by the sum of $f_{1, A_{k 1}^{(i+1)}}$ from the Stage-1 frequency set $\mathbf{F}_{1}, f_{2, A_{k 2}^{(i+1)}}$ from the Stage-2 frequency set $\mathbf{F}_{2}$, etc. until $f_{S, A_{k S}^{(i+1)}}$ from the Stage- $S$ frequency set $\mathbf{F}_{S}$, yielding $\sum_{s=1}^{S} f_{s, A_{k s}^{(i+1)}}$. The above procedure is continued until the end of transmission of user $k$. Since the dynamic range of the above RNS is $\left[0, M_{s}=\prod_{s=1}^{S} m_{s}\right)$, the period of the FH patterns is $M_{S}$.

Multi-user FHMA: Let us now discuss the RNS-based MS/UWB FHMA scheme. Let $m_{1}, m_{2}, \ldots, m_{S}$ be the pairwise relative primes representing the number of frequencies in the $S$ number of frequency sets, namely in $\mathbf{F}_{1}, \mathbf{F}_{2}, \ldots, \mathbf{F}_{S}$, with respect to the $S$ FH stages of Fig.2, respectively. Let $\mathbf{A}_{k} \Leftrightarrow\left(A_{k 1}, A_{k 2}, \ldots, A_{k S}\right)$, where $0 \leq \mathbf{A}_{k}<M_{S}$, and $\mathbf{a}_{k}=\left[a_{k}(0), a_{k}(1), \ldots, a_{k}(L-1)\right]$ be the FH address and MUD address of user $k$, respectively. Furthermore, let $\mathbf{C} \Leftrightarrow$ $\left(C_{1}, C_{2}, \ldots, C_{S}\right)$ represent a reference register in the RNS domain, which provides reference to each user, in order to implement synchronous transmission. $\mathbf{C}$ is increased by one, whenever $S$ hops happened. According to (3), this can be implemented by increasing each element of $\left(C_{1}, C_{2}, \ldots, C_{S}\right)$ by one with respect to their corresponding modulus. Note that although the maximum integer represented by the RNS-based registers $\left(C_{1}, C_{2}, \ldots, C_{S}\right)$ is $M_{S}-1=\prod_{s=1}^{S} m_{s}-1$, this will not prevent us from using it as a synchronous transmission reference. Indeed, this property can substantially simplify the implementation of synchronous transmissions, as we will show below. According to the above assumptions, when the $k$ th user is attempting to access the network and to transmit the information symbol $X_{k}^{(m)}$, the operation of the multistage FHMA scheme can be described with the aid of the following steps:

1) According to (1), $X_{k}^{(m)}$ is signalled with the aid of the $k$ th user's MUD address with respect to the chips $0,1, \ldots, L-1$, where $L=\omega S$. The $M$-ary symbols are expressed as $\mathbf{Y}_{k}=\left[y_{k}(0), y_{k}(1), \ldots, y_{k}(L-1)\right]=$ $X_{k}^{(m)} \cdot \mathbf{1} \bigoplus \mathbf{a}_{k}$, which are signalled using the $L$ number of chips each hosting one of the $M$ possible signalling tones.

2) Based on the $k$ th user's FH address $\mathbf{A}_{k}$ and on the reference register's instantaneous value of $\mathbf{C}$, we derive the residue sequence $\mathbf{I}_{k}$ as follows:

$$
\mathbf{I}_{k} \Leftrightarrow\left(i_{1}, i_{2}, \ldots, i_{S}\right)=\left[\left(A_{k s} \oplus C_{s}\right)\left(\bmod m_{s}\right)\right]_{s=1}^{S},
$$

according to (3). 
3) For the chip $t S \leq l<(t+1) S, t \leq \omega-1$, the transmitted frequency is constituted by the sum of the frequencies $f_{1, i_{1}+t}$ from the Stage- 1 frequency set $\mathbf{F}_{1}, f_{2, i_{2}+t}$ from the Stage-2 frequency set $\mathbf{F}_{2}$, etc. upto $f_{S-(l-t S)-1, i_{S-(l-t S)-1}+t}$ from the Stage- $(S-(l-$ $t S)-1)$ frequency set $\mathbf{F}_{S-(l-t S)-1}$, and the frequencies $f_{S-(l-t S), i_{S-(l-t S)}+t+1}$ from the Stage- $(S-(l-t S))$ frequency set $\mathbf{F}_{S-(l-t S)}$ upto $f_{S, i_{S}+t+1}$ from the Stage$S$ frequency set $\mathbf{F}_{S}$, as well as the frequency $f_{d, l}^{(k)}$ corresponding to the MFSK tone activated according to $y_{k}(l)$. The frequency given by the corresponding sum can be expressed as

$$
f_{k, l}=\sum_{j=1}^{S-(l-t S)-1} f_{j, i_{j}+t}+\sum_{j=S-(l-t S)}^{S} f_{j, i_{j}+t+1}+f_{d, l}^{(k)}
$$

4) The remaining MFSK symbols are transmitted by repeating the above three steps.

According to the above principles, if the number of users satisfies $K \leq M_{s}, K$ out of the $M_{s} \mathrm{FH}$ addresses, which are expressed in the form of residue sequences derived from the integers $0,1, \ldots, M_{s}-1$, can be uniquely assigned to the $K$ users. Again, based on this unique assignment and on the RNSbased FHMA scheme described above, it can be readily shown that no collisions occur between any two users, since at any moment, the addition of these FH addresses to the same instantaneous value of the common RNS-based reference register will generate $K \leq M_{s}$ different integers in the range of $\left[0, M_{s}\right)$. Consequently, the hopping frequencies derived from the resultant $K$ integers represented as $K$ residue sequences are unique. Therefore, again, if the number of users obeys $K \leq M_{s}$, the probability of hits or collisions is zero.

However, the MS/UWB FHMA system is capable of supporting more than $K$ users, if efficient MUD techniques can be employed. More explicitly, the $S$-stage MS/UWB FHMA system using the RNS moduli $\left(m_{1}, m_{2}, \ldots, m_{S}\right)$ and MFSK modulation can support upto $K=M \times \prod_{s=1}^{S} m_{s}$ number of users by invoking multiuser detection techniques [7], [8]. If the number of users, $K$, is higher than the number of unique FH addresses, $M_{S}$, i.e., when $K>M_{S}$, then a given $\mathrm{FH}$ address can be assigned to a group of users, and the users in this group are distinguished by their corresponding MUD address, $\mathbf{a}_{k}=\left[a_{k}(0), a_{k}(1), \ldots, a_{k}(L-1)\right], k=1,2, \ldots, K$. More explicitly, if $U<K / M_{s} \leq U+1 \leq M$, then a given $\mathrm{FH}$ address has to be assigned to more than one, namely to $U$ or $U+1$ users, which form a user group. In order to resolve the associated ambiguity, an additional set of $U$ or $(U+1)$ MUD addresses are assigned employing a unique mapping to each of the $U$ or the $(U+1)$ users in the same user group, in order to distinguish them from each other. Note that, the same MUD address can be assigned to several users belonging to different groups, since the groups are differentiated with the aid of the unique $\mathrm{FH}$ addresses. Based on the above user signature assignment rule, if non-fading channels or frequency non-selective fading channels are encountered by each MFSK tone, then the

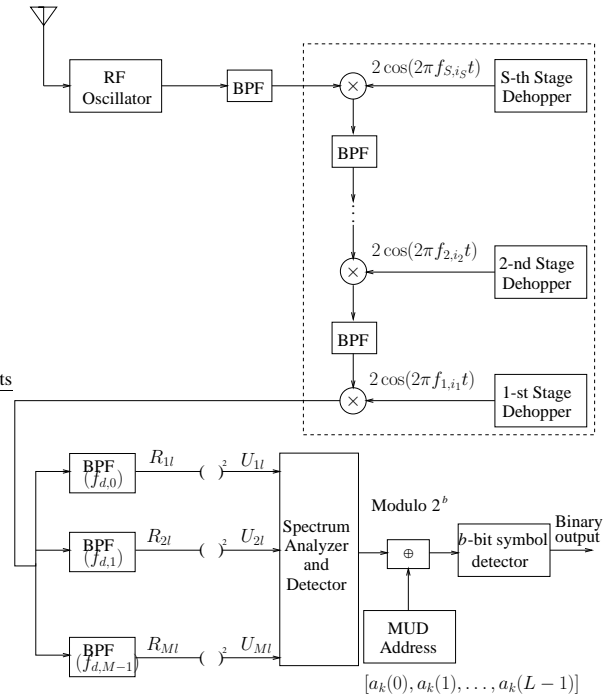

Fig. 3. Receiver block diagram of the MS/UWB FH SSMA system using MFSK modulation.

multiuser detector of the synchronous MS/UWB FHMA system only has to differentiate amongst the users of the same group, i.e. the users that were assigned the same FH address. Consequently, the number of users detected by the multiuser receiver is about $K / M_{s}$, which is not higher than $M$, and significantly lower than $K$ representing the total number of users supported by the MS/UWB FHMA system. Therefore, the complexity of the multiuser receiver employed in the MS/UWB FHMA system can be substantially decreased in comparison to that of the conventional MUD concept, where all system users are detected simultaneously.

Above we have discussed the transmitter, the spectrum assignment, the FH strategy as well as the achievable diversity order of the proposed MS/UWB FHMA system. Let us now consider the detection of the MS/UWB FHMA signals.

\section{DETECTION OF MS/UWB FHMA SIGNALS}

\section{A. Receiver Description}

The receiver schematic of the MS/UWB FHMA system is shown in Fig.3, which essentially follows the inverse structure of the transmitter. At the receiver the received signal is first down-converted to the Intermediate Frequency (IF) corresponding to the FH pattern used. Then, the down-converted signal is frequency dehopped as indicated by the dashed-line box of Fig.3. In contrast to the FH scheme of the transmitter in Fig.1, where FH commences at Stage- 1 and continues to the $S$ th stage, at the receiver of Fig. 3 the hopping frequencies are removed in the order commencing with the $S$ th stage and finishing at the 1st stage, according to the specific FH pattern of a given user. Following the frequency de-hopping stage, the received signal is input to a bank of $M$ energy detectors matched to the $M$ frequency tones of the MFSK stage. The detection interval is $T_{h}$ seconds, which is synchronized with the chips or FH intervals of the desired user. The $M$ energy detectors provide $M L$ outputs during a symbol period of $T_{s}$ seconds. As shown in Fig.3, 


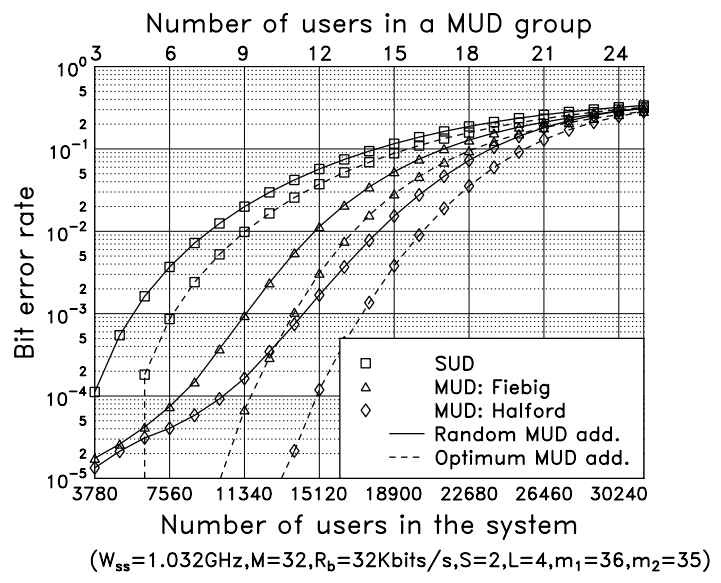

Fig. 4. Bit error rate shown for the conventional single-user detector (SUD), for Fiebig's [7] interference cancellation multiuser detector (MUD: Fiebig), and for the multiuser detector based on multistage interference cancellation [8] (MUD: Halford) in the context of a 2-stage UWB FHMA system associated with parameters $m_{1}=36, m_{2}=35, M=32, S=2$ and $L=4$ for transmission over noiseless interference-contaminated channels $\left(E_{b} / N_{0}=\infty\right)$.

the spectrum of each $T_{s}$-duration transmission is analyzed in order to determine, which frequency tone and hence which $b$-bit symbol $X_{k}^{(m)}$ was transmitted by invoking the MUD address of the desired user.

\section{B. Performance Example}

The BER performance of the MS/UWB FHMA system is studied by simulation for three detection schemes, namely for the conventional Single-User Detector (SUD), the MUD proposed by Fiebig [7] (MUD: Fiebig) and the MUD proposed by Halford and Brandt-Pearce (MUD: Halford). In our simulations, two types of MUD address are considered, namely a random and the optimum MUD address assignment. In the context of the random MUD address assignment scheme, each user's MUD address is randomly generated, and the $k$ th user's MUD address can be expressed as

$$
\mathbf{a}_{k}=\left(\gamma_{k}, \gamma_{k} \beta, \gamma_{k} \beta^{2}, \ldots, \gamma_{k} \beta^{L-1}\right)
$$

where $\gamma_{k}$ is an element of $G F(M)$ assigned to user $k$ and $\beta$ is a fixed primitive element of $G F(M)$. In our simulations $G F(32)$ was considered and the corresponding primitive polynomial was $p(x)=1+x^{2}+x^{5}$.

Fig.4 and Fig.5 present the BER comparison of the abovementioned three detection schemes, when there is no additive channel noise $\left(E_{b} / N_{0}=\infty\right)$. The parameters used in our simulations were listed in the related figures. As shown in the figures, we assumed that the total system bandwidth was 1.032GHz in Fig. 4 and 1.22GHz in Fig.5. The results show that after the 1st stage interference cancellation (IC), the multiuser detectors using both iterative IC [7] and multistage IC [8] outperform the conventional single-user detector by a wide margin. The optimum MUD address assignment significantly outperformed the random MUD address assignment in the context of

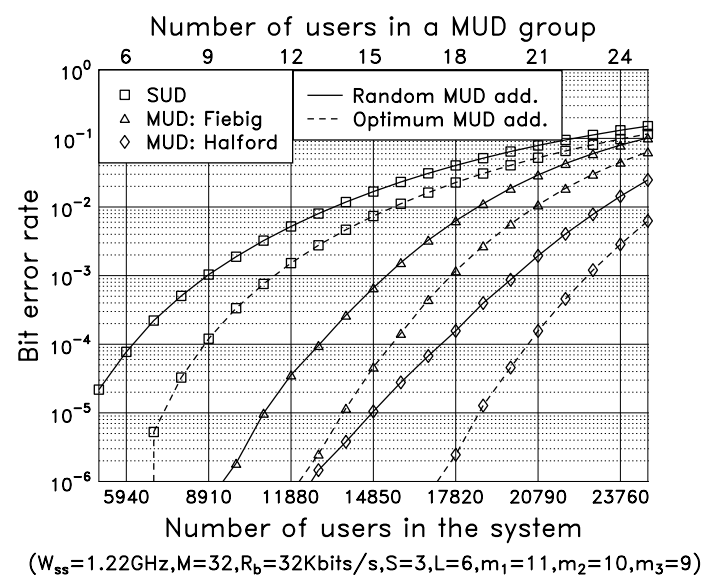

Fig. 5. Bit error rate shown for the conventional single-user detector (SUD), for Fiebig's [7] interference cancellation multiuser detector (MUD: Fiebig), and for the multiuser detector based on multistage interference cancellation [8] (MUD: Halford) in the context of a 3-stage UWB FHMA system associated with parameters $m_{1}=11, m_{2}=10, m_{3}=9, M=32, S=3$ and $L=6$ for transmission over noiseless interference-contaminated channels $\left(E_{b} / N_{0}=\infty\right)$

all three detection schemes. Furthermore, for the noiseless but interference-contaminated channel considered, the MUD employing multistage IC [8] outperforms iterative IC [7]. Note that although the total number of users supported by the MS/UWB FHMA system may be as high as tens of thousands, the number of users to be detected by each multiuser detector is extremely low, as shown at the top of Fig.4. Therefore, the MS/UWB FHMA system is capable of supporting a tremendous number of users, while employing low-complexity receivers.

\section{REFERENCES}

[1] M. Z. Win, R. A. Scholtz and M. A. Barnes, "Ultra-wide bandwidth signal propogation for indoor wireless communications," Proc. IEEE Int. Conf. on Commun., Vol. 1, pp. 56-60, Montreal, Canada, June 1997.

[2] M. Z. Win and R. A. Scholtz, "On the robustness of ultra-wide bandwidth signals in dense multipath environments" IEEE Commun. Lett., vol. 2, no. 2, pp. 51-53, Feb. 1998

[3] M. Z. Win and R. A. Scholtz, "Energy capture vs. correlator resources in ultra-wide bandwidth indoor wireless communications channels" Proc. Military Comm. Conf., vol. 3, pp. 1277-1281, Monterey, Canada, Nov. 1997.

[4] M. Z. Win and R. A. Scholtz, "On the energy capture of ultra-wide bandwidth signals in dense multipath environments" IEEE Commun. Lett., vol. 2, no. 9 , pp. 245-247, Sept. 1998.

[5] M. Z. Win and R. A. Scholtz, "Ultra-wide bandwidth time-hopping spread-spectrum impulse radio for wireless multiple-access communications," IEEE Trans. on Commun., vol. 48, pp. 679-691, April 2000.

[6] D. J. Goodman, P. S. Henry, and V. K. Prabhu, "Frequency-hopped multilevel FSK for mobile radio," Bell System Technical Journal, vol. 59, pp. 1257-1275, Sept. 1980.

[7] U. C. G. Fiebig, "Iterative interference cancellation for FFH/MFSK MA systems," IEE Proc. Commun., vol. 143, pp. 380-388, Dec. 1996.

[8] K. W. Halford and M. Brandt-Pearce, "Multistage multiuser detection for FHMA,” IEEE Trans. on Commun., vol. 48, pp. 1550-1562, Sept. 2000.

[9] R. W. Watson and C. W. Hastings, "Self-checked computation using residue arithmetic," Proc. of the IEEE, vol. 54, pp. 1920-1931, Dec. 1966. 